\title{
Risk of Post-TKA Acute Myocardial Infarction in Patients With a History of Myocardial Infarction or Coronary Stent
}

\author{
Atul Kumar MBBS, DRE, Wen-Chen Tsai DrPH, Tai-Sheng Tan MD, \\ Li-Ting Chiu MHA, Pei-Tseng Kung ScD, Ching-Kan Lo MD, \\ Ming-Chou Ku MD
}

Received: 19 July 2015/ Accepted: 26 October 2015/Published online: 13 November 2015

(C) The Association of Bone and Joint Surgeons \& 2015

\begin{abstract}
Background Acute myocardial infarction (AMI) is one of the most important perioperative complications of total knee arthroplasty (TKA). Although risk-stratification tools exist for the prediction of cardiac complications including AMI after noncardiac surgery, such stratification does not differentiate the patients with a coronary stent alone, AMI
\end{abstract}

Each author certifies that he or she, or a member of his or her immediate family, has no funding or commercial associations (eg, consultancies, stock ownership, equity interest, patent/licensing arrangements, etc) that might pose a conflict of interest in connection with the submitted article.

All ICMJE Conflict of Interest Forms for authors and Clinical Orthopaedics and Related Research ${ }^{\mathbb{R}}$ editors and board members are on file with the publication and can be viewed on request.

Each author certifies that his or her institution approved the human protocol for this investigation and that all investigations were conducted in conformity with ethical principles of research. This work was performed at Chang Bing Show Chwan Memorial Hospital, Changhua, Taiwan, Republic of China.

Authors W.-C. Tsai and M.-C. Ku made equal contributions to this work.

A. Kumar, T.-S. Tan

Chang Bing Show Chwan Memorial Hospital, Changhua,

Taiwan, Republic of China

A. Kumar

IRCAD-Taiwan, Changhua, Taiwan, Republic of China

C.-K. Lo, M.-C. Ku ( $₫)$

Show Chwan Memorial Hospital, 542, Sec 1 Chung-shan Road, Changhua County 505, Taiwan, Republic of China

e-mail: showjoeku@gmail.com

P.-T. Kung

Asia University, Taichung, Taiwan, Republic of China

W.-C. Tsai, L.-T. Chiu

China Medical University, Taichung, Taiwan, Republic of China without a stent, or AMI with a stent. The risk of postoperative AMI in these patient groups may vary. Several studies have recommended suitable times for noncardiac surgery in patients with a coronary stent; however, they do not differentiate between the patients with AMI and no AMI. The suitable time of noncardiac surgery for patients with AMI and stent may vary from those with a stent alone. Moreover, a study to evaluate the risk of AMI within 1 year in an Asian population with a history of AMI or coronary stent who underwent TKA has not been reported.

Questions/purposes (1) What are the risks of AMI within 1 year of TKA in patients who have had a stent alone, AMI without a stent, or AMI with a stent as compared with patients without an AMI/stent? (2) For patients with AMI/ stent placement, when can TKA be performed where the risk of subsequent AMI normalizes? (3) What comorbidities are associated with post-TKA AMI? (4) Is the risk of AMI within 1 year after surgery in patients undergoing TKA without a history of AMI/stent higher than that in patients with no surgery?

Methods This study is a retrospective study of the medical claim records of 128,216 patients who underwent TKA between 1997 and 2010 in Taiwan. The records were retrieved from the research database of the Bureau of National Health Insurance in Taiwan, which maintains the records of $99.68 \%$ of the Taiwan population. The patients who had a history of AMI or coronary stent placement within the year before TKA were compared with the patients who had not experienced AMI or stent placement before TKA. The control subjects were matched according to sex, age, Charlson score, and year of surgery. There were 2413 patients in each group. The patients with a history of AMI or stent placement and the timing of TKA after coronary event were further stratified as with a coronary stent alone, AMI without a stent, and AMI with a 
stent. The effects of the comorbidities of renal failure, diabetes, liver failure, and hypertension were also analyzed individually. The risk of AMI within 1 year after TKA was investigated using bivariate analysis and the Cox proportional hazard model. To compare the risk of AMI within 1 year of surgery in the patients with a history of TKA and no $\mathrm{AMI} /$ stent with the population without a history of surgery, a similar bivariate analysis and the Cox proportional hazard model were applied to their matched case and control groups, each containing 110,980 patients.

Results In the adjusted model, using no AMI/stent before TKA as a reference, patients having undergone AMI + stent had the highest risk (hazard ratio [HR], 5.23; 95\% confidence interval $[\mathrm{CI}], 1.81-15.14 ; \mathrm{p}=0.002)$, AMI alone without a stent had less risk (HR, 4.88; 95\% CI, $1.49-16.01 ; p=0.009$ ), and stent alone with AMI had the lowest risk (HR, 3.16; 95\% CI, 1.29-7.71; $p=0.012$ ). In all patients, risk of AMI after TKA was not different than reference values after 1 year of initial AMI or stent (stent: HR, 1.67; 95\% CI, 0.71-3.94; $\mathrm{p}=0.239$; AMI: HR, 1.88; 95\% CI, 0.42-8.49; $\mathrm{p}=0.412$; AMI + stent: HR, 1.91; $95 \% \mathrm{CI}, 0.53-6.89 ; \mathrm{p}=0.321$ ). The risk of post-TKA AMI was elevated within 1 year of the previous episode of AMI/ stent (0-180 days: HR, 8.42; 95\% CI, 3.03-23.41; p < 0.001; 181-365 days: HR, 7.52; 95\% CI, 2.47-22.88; $\mathrm{p}<$ 0.001 ). Only chronic renal failure under hemodialysis was associated with increased risk of AMI within 1 year of TKA (adjusted HR, 4.34; 95\% CI, 1.22-15.43; $\mathrm{p}=0.023$ ). Patients undergoing TKA with no history of AMI/stent had a lower risk of AMI within 1 year of TKA compared with the patients with no history of surgery (adjusted HR, 0.92; 95\% CI, 0.86-0.99; $\mathrm{p}=0.016$ ).

Conclusions This study found the risk of post-TKA AMI remains high within 1 year in patients with a history of AMI/stent. It is recommended that an elective TKA should be performed at least 1 year after an episode of AMI or stent placement. Stents do not provide protection against post-TKA AMI within 6 months of the AMI and patients with AMI + stent have a higher risk of AMI than those with only AMI. Patients of AMI/stent on hemodialysis have a very high risk of post-TKA AMI. However, the risk of AMI is lower in post-TKA patients compared with those with no TKA.

Level of Evidence Level III, prognostic study.

\section{Introduction}

Acute myocardial infarction (AMI) after TKA is a potentially devastating complication, resulting in death in $12 \%$ to $25 \%$ of patients who experience it $[1,4,22]$. Patients undergoing TKA who have had a previous AMI are at increased risk of having an AMI after the knee procedure
[12]. Some studies have reported $0.4 \%$ to $1.8 \%$ of patients experience myocardial infarction after TKA [8, 15]. A retrospective study from a health institute in Canada cautioned readers against hospital discharge of patients within 3 days of the TKA [6]. Furthermore, a study on a northern Illinois population of primary TKA reported cardiac complications as one of the major concerns among all the complications [5]. Moreover, cardiovascular disease was reported as a major risk factor for death and myocardial infarction in a study to determine preoperative anemia as an independent risk factor for 30-day mortality and myocardial infarction after TKA [16].

The majority of such studies have evaluated the incidence of AMI after TKA focusing on the short-term period after the surgery (up to 90 days). Only one study on a Danish population has reported the risk of AMI within 1 year after total joint arthroplasty. This study also investigated the time interval between TKA and postsurgical AMI in patients with a history of AMI or coronary stent placement. The study reported that the AMI risk after TKA is increased during the first 2 weeks of surgery and does not differ from the risk resulting from other noncardiac surgery after the first 2 weeks. To our knowledge, no such study has been reported in an Asian population. Risk-stratification tools, from the American College of Cardiology (ACA)/American Heart Association (AHA), exist for the prediction of cardiac complications including AMI after noncardiac surgery [6], which can be applied to individual patients in a prospective way to estimate their cardiac risk. However, such stratification does not differentiate the patients with a coronary stent alone, AMI without a stent, or AMI with a stent. The knowledge of the risk specific to patients with a history of AMI and/or stent placement would further guide surgeons in deciding when to perform an elective TKA in those patients. Although the guidelines suggest a risk for AMI after noncardiac surgery, they do not indicate if the stent in patients with AMI reduces the risk of post-TKA AMI to an extent that would allow a TKA to be performed within 1 year. The goal of this study was to evaluate the risk of AMI within 1 year in patients who underwent TKA and to find a suitable time for TKA in patients who have experienced a previous episode of AMI or coronary stenting. The study differs from other previous studies in the following ways: (1) it emphasizes the interval between the previous episode of AMI or stent placement and the TKA; and (2) it includes comorbidities such as hypertension, liver failure, and renal failure, which were not considered in other previous studies.

We therefore asked: (1) What are the risks of AMI within 1 year of TKA in patients who have had a stent alone, AMI without a stent, or AMI with a stent as compared with patients without AMI/stent? (2) For patients with $\mathrm{AMI} /$ stent placement, when can TKA be performed 
where the risk of subsequent AMI normalizes? (3) What comorbidities are associated with post-TKA AMI? (4) Is the risk of AMI within 1 year after surgery in patients undergoing TKA without a history or AMI/stent higher than that in patients with no surgery?

\section{Patients and Methods}

This study is a retrospective case-control study of the medical claim records of patients who underwent TKA between 1997 and 2010 in Taiwan. The data were retrieved from the research database of the Bureau of National Health Insurance (BNHI) in Taiwan. BNHI is a government-owned agency responsible for providing healthcare services, reviewing medical fees, and the suitability of such services provided to the Taiwan population. The BNHI database maintains a nationwide database covering $99.68 \%$ of the Taiwan population [17]. The database has been reported to have up to $94 \%$ accuracy $[2,3]$. Moreover, several hundred publications have been based on the data. However, medical records of individual patients in the database lack information such as laboratory investigation reports and the particular limb that underwent surgery. The study on the BNHI data was approved by the research ethics committee in China Medical University and Hospital, Taiwan.

$\mathrm{BNHI}$ procedural/diagnostic codes were used to retrieve data of TKA, stent, and AMI patients. The codes correspond to the International Classification of Diseases, 9th Revision procedure codes (ICD-9) for TKA (8.54), stent (36.01, 36.02, 36.05), and AMI (410.00, 410.92). The patients who underwent TKA between 1997 and 2010 were included in the study. The study included only primary TKA performed in Taiwan. It did not include revision TKA or tumor prosthesis reconstruction for bone tumors.

\section{Comparison Between Patients With AMI/Stent and Without AMI/Stent}

Propensity score matching was adopted to mitigate the differences between the patients who had a history of AMI or coronary stent placement before TKA and the patients who had not experienced AMI or stent placement before TKA (Table 1). The 4826 patients were equally allocated to the two groups (2413 in each group) based on their sex, age, Charlson Comorbidity Index, and the year of surgery. After matching, the two groups showed no difference $(\mathrm{p}=1.00)$.

A total of 128,216 patients who had undergone TKA were included in the study. After the propensity score matching, 4826 patients (2413 with a history of AMI or coronary stent placement and 2413 with no such history) were studied further to investigate the importance of various variables in the occurrence of AMI after TKA. The patients with a history of AMI or coronary stent placement had a similar distribution of age (mean age, 73 years) and sex (55\%:45\%, female:male) compared with those of the matched control subjects. A bivariate analysis for the percentage of post-TKA AMI with respect to various independent variables and calculation of the Cox proportional hazards ratio to investigate the related risks of a postTKA AMI were carried out.

In addition to dividing patients into groups based on history of AMI or coronary stent placement before TKA (stent, AMI, AMI + stent, and no AMI/stent), the study used the period between an AMI episode or stent placement before TKA to divide the patients into different groups (0-180 days, 181-365 days, $>365$ days, no AMI/stent). Demographic data for the patients were sex and age. The health status variables comprised the existence of major diseases and comorbidities (such as end-stage renal disease under hemodialysis, hepatitis $\mathrm{B}$ and $\mathrm{C}$, cirrhosis, diabetes, and hypertension). Patients were considered as having a comorbidity if they visited a clinic three times or were hospitalized once or more for any comorbidity in the 3 years before undergoing TKA.

\section{Statistical Analysis}

SAS statistical software (Version 9.3 for Windows; SAS Institute, Inc, Cary, NC, USA) was used for the data processing and statistical analysis. A descriptive statistical analysis of the variables such as TKA patients' personal characteristics and health status with chi-square test and ttest were used to compare the differences in the variables between post-TKA patients who experienced an AMI within 1 year and those who did not.

A Cox proportional hazards model was used to study the related risks of AMI in patients undergoing TKA within 1 year after surgery controlling for relevant variables. Patients who experienced an AMI within 1 year after TKA were defined as "post-TKA AMI," whereas those who did not experience an AMI within 1 year after TKA were defined as "censored". Independent variables were AMI episode or coronary stent placement before the TKA, period between AMI episode or stent placement and TKA, patients' demographic characteristics (ie, sex and age), health status (ie, major diseases and comorbidities), and the calendar year in which the TKA was performed. The dependent variable was AMI episode within 1 year after TKA. A stratified bivariate analysis for the time interval between TKA and the previous event of AMI/stent placement was also evaluated. All the variables in the bivariate analysis were also used in the Cox analysis. The Cox analysis was done with three models: one unadjusted model and two (A and $\mathrm{B}$ ) adjusted models. In the 
Table 1. Comparisons of patients undergoing TKA with AMI/stent and without AMI/stent before and after propensity score matching

\begin{tabular}{|c|c|c|c|c|c|c|c|c|c|c|}
\hline \multirow[t]{4}{*}{ Variables } & \multicolumn{5}{|c|}{ Before matching } & \multicolumn{5}{|c|}{ After matching } \\
\hline & \multirow{2}{*}{\multicolumn{2}{|c|}{$\frac{\text { No AMI/stent before TKR }}{\left(\mathrm{N}_{1}=12,5803\right)}$}} & \multirow{2}{*}{\multicolumn{2}{|c|}{$\frac{\text { AMI/stent before TKR }}{\left(\mathrm{N}_{2}=2413\right)}$}} & \multirow[t]{3}{*}{$\mathrm{p}$ value } & \multirow{2}{*}{\multicolumn{2}{|c|}{$\begin{array}{l}\text { No AMI/stent before TKR } \\
\left(\mathrm{N}_{1}=2413\right) \\
\end{array}$}} & \multirow{2}{*}{\multicolumn{2}{|c|}{$\begin{array}{l}\text { AMI/stent before TKR } \\
\left(\mathrm{N}_{2}=2413\right) \\
\end{array}$}} & \multirow[t]{3}{*}{$\mathrm{p}$ value } \\
\hline & & & & & & & & & & \\
\hline & $\mathrm{N}_{1}$ & Percent & $\mathrm{N}_{2}$ & Percent & & $\mathrm{N}_{1}$ & Percent & $\mathrm{N}_{2}$ & Percent & \\
\hline Gender & & & & & $<0.001$ & & & & & 1.000 \\
\hline Female & 93,418 & 74.26 & 1329 & 55.08 & & 1329 & 55.08 & 1329 & 55.08 & \\
\hline Male & 32,385 & 25.74 & 1084 & 44.92 & & 1084 & 44.92 & 1084 & 44.92 & \\
\hline Age (years) & & & & & $<0.001$ & & & & & 1.000 \\
\hline$<65$ years & 33,663 & 26.76 & 322 & 13.34 & & 322 & 13.34 & 322 & 13.34 & \\
\hline $65-69$ & 28,430 & 22.60 & 444 & 18.40 & & 444 & 18.40 & 444 & 18.40 & \\
\hline $70-74$ & 31,738 & 25.23 & 680 & 28.18 & & 680 & 28.18 & 680 & 28.18 & \\
\hline $75-79$ & 21,789 & 17.32 & 589 & 24.41 & & 589 & 24.41 & 589 & 24.41 & \\
\hline$\geqq 80$ & 10,183 & 8.09 & 378 & 15.67 & & 378 & 15.67 & 378 & 15.67 & \\
\hline $\mathrm{CCI}$ & & & & & $<0.001$ & & & & & 1.000 \\
\hline 0 & 19,954 & 15.86 & 158 & 6.55 & & 158 & 6.55 & 158 & 6.55 & \\
\hline 1 & 25,718 & 20.44 & 373 & 15.46 & & 373 & 15.46 & 373 & 15.46 & \\
\hline 2 & 23,195 & 18.44 & 478 & 19.81 & & 478 & 19.81 & 478 & 19.81 & \\
\hline 3 & 17,285 & 13.74 & 369 & 15.29 & & 369 & 15.29 & 369 & 15.29 & \\
\hline 4 & 12,524 & 9.96 & 326 & 13.51 & & 326 & 13.51 & 326 & 13.51 & \\
\hline$\geqq 5$ & 27,127 & 21.56 & 709 & 29.38 & & 709 & 29.38 & 709 & 29.38 & \\
\hline Surgery year & & & & & $<0.001$ & & & & & 1.000 \\
\hline 1998-2001 & 27,382 & 21.77 & 198 & 8.21 & & 198 & 8.21 & 198 & 8.21 & \\
\hline 2002-2005 & 34,845 & 27.70 & 500 & 20.72 & & 500 & 20.72 & 500 & 20.72 & \\
\hline 2006-2010 & 63,576 & 50.54 & 1715 & 71.07 & & 1715 & 71.07 & 1715 & 71.07 & \\
\hline
\end{tabular}

$\mathrm{AMI}=$ acute myocardial infarction; $\mathrm{CCI}=$ Charlson Comorbidity Index.

unadjusted model, each variable was examined individually for its relationship with the dependent variable (postTKA AMI) without other variables being included in the model. Models A and B were the adjusted models, which included all relevant variables in the model to be analyzed at the same time and investigated the independent effect of each variable on the post-TKA AMI when all other relevant variables in the model are controlled. The difference between Model A and Model B was the key independent variable. In Model A, the key independent variable was whether the patients undergoing TKA had a previous episode of AMI or went through coronary stent placement, and in Model B, it was the time interval between the AMI or stent placement and the TKA. A stratified hazard ratio analysis for the time interval between TKA and previous $\mathrm{AMI} /$ stent placement was also evaluated.

\section{Comparison Between Patients Undergoing TKA and Non-TKA Patients}

A propensity score matching was also performed to mitigate the difference between the patients who had TKA with no history of AMI/stent and the patients who had no history of surgery (Table 2). This comprised of 221,960 patients (110,980 patients with TKA and 110,980 patients with no surgery). The risk of AMI within 1 year after TKA in the patients with no history of AMI/stent was compared with that in patients with no history of surgery using a calculation of the Cox proportional hazards ratio.

\section{Results}

All groups with AMI/stent had elevated risk of post-TKA AMI compared with that of the reference with no AMI/ stent within 1 year of $\mathrm{AMI} /$ stent. In the adjusted model (Table 3), using no AMI/stent before TKA as the reference, patients having undergone AMI + stent had the highest risk (hazard ratio [HR], 5.23; 95\% confidence interval [CI], 1.81-15.14; $\mathrm{p}=0.002)$, AMI alone without stent had less risk (HR, 4.88; 95\% CI, 1.49-16.01; $\mathrm{p}=0.009$ ), and stent alone with AMI had the lowest risk (HR, 3.16; 95\% CI, 1.29-7.71; $\mathrm{p}=0.012)$. The overall rate of AMI in the study population was $0.77 \%$. The patients with AMI without a stent had the highest percentage of post-TKA AMI (stent: 
Table 2. Comparisons of patients undergoing TKA and non-TKA patients before and after propensity score matching

\begin{tabular}{|c|c|c|c|c|c|c|c|c|c|c|}
\hline \multirow[t]{3}{*}{ Variables } & \multicolumn{5}{|c|}{ Before matching } & \multicolumn{5}{|c|}{ After matching } \\
\hline & \multicolumn{2}{|c|}{ Non-TKA $\left(\mathrm{N}_{1}=968,497\right)$} & \multicolumn{2}{|c|}{ TKA $\left(\mathrm{N}_{2}=125,803\right)$} & \multirow[t]{2}{*}{$\mathrm{p}$ value } & \multicolumn{2}{|c|}{ Non-TKA $\left(\mathrm{N}_{1}=110,980\right)$} & \multicolumn{2}{|c|}{ TKA $\left(\mathrm{N}_{2}=110,980\right)$} & \multirow[t]{2}{*}{$\mathrm{p}$ value } \\
\hline & $\mathrm{N}_{1}$ & Percent & $\mathrm{N}_{2}$ & Percent & & $\mathrm{N}_{1}$ & Percent & $\mathrm{N}_{2}$ & Percent & \\
\hline Gender & & & & & $<0.001$ & & & & & 1.000 \\
\hline Female & 470,482 & 48.58 & 93,418 & 74.26 & & 78,617 & 70.84 & 78,617 & 70.84 & \\
\hline Male & 498,015 & 51.42 & 32,385 & 25.74 & & 32,363 & 29.16 & 32,363 & 29.16 & \\
\hline Age (years) & & & & & $<0.001$ & & & & & 0.574 \\
\hline$<65$ & 892,881 & 92.19 & 33,663 & 26.76 & & 33,641 & 30.31 & 33,641 & 30.31 & \\
\hline $65-69$ & 27,010 & 2.79 & 28,430 & 22.60 & & 28,652 & 25.82 & 28,418 & 25.61 & \\
\hline $70-74$ & 22,519 & 2.33 & 31,738 & 25.23 & & 25,174 & 22.68 & 25,174 & 22.68 & \\
\hline $75-79$ & 14,204 & 1.47 & 21,789 & 17.32 & & 13,942 & 12.56 & 14,176 & 12.77 & \\
\hline$\geqq 84$ & 11,883 & 1.23 & 10,183 & 8.09 & & 9571 & 8.62 & 9571 & 8.62 & \\
\hline CCI & & & & & $<0.001$ & & & & & 0.663 \\
\hline 0 & 436,934 & 45.11 & 19,954 & 15.86 & & 19,015 & 17.13 & 19,249 & 17.34 & \\
\hline 1 & 192,848 & 19.91 & 25,718 & 20.44 & & 22,956 & 20.68 & 22,956 & 20.68 & \\
\hline 2 & 111,652 & 11.53 & 23,195 & 18.44 & & 19,397 & 17.48 & 19,397 & 17.48 & \\
\hline 3 & 69,162 & 7.14 & 17,285 & 13.74 & & 14,495 & 13.06 & 14,267 & 12.86 & \\
\hline 4 & 44,943 & 4.64 & 12,524 & 9.96 & & 10,448 & 9.41 & 10,448 & 9.41 & \\
\hline$\geqq 5$ & 112,958 & 11.66 & 27,127 & 21.56 & & 24,669 & 22.23 & 24,663 & 22.22 & \\
\hline
\end{tabular}

$\mathrm{CCI}=$ Charlson Comorbidity Index.

1.05\%; AMI: $1.77 \%$; AMI + stent: $1.66 \%$, no AMI/stent: $0.29, \mathrm{p}=0.001)$.

In all patients, risk of AMI after TKA was not different than reference values (Table 4 ) after 1 year of the initial AMI or stent (stent: HR, 1.67; 95\% CI, 0.71-3.94; $\mathrm{p}=0.239$; AMI: HR, 1.88; 95\% CI, 0.42-8.49; $\mathrm{p}=0.412$; AMI + stent: HR, 1.91; 95\% CI, 0.53-6.89; $\mathrm{p}=0.321)$. However, all patients had a higher risk of AMI after TKA (stent: HR, 4.98; 95\% CI, 1.72-14.38; $\mathrm{p}=0.003$; AMI: HR, 16.11; 95\% CI, 1.96-132.22; $\mathrm{p}=0.010$; AMI + stent: HR, 27.27; 95\% CI, 7.52-98.94; $\mathrm{p}<0.001)$ in the 180 days after the initial event. Among the patients operated on within 180 days of an AMI/stent, patients with AMI + stent placement had the highest risk of post-TKA AMI. Within 1 year of AMI (with or without stent), patients had a high risk of post-TKA AMI (<180 days: HR, 8.54; 95\% CI, 3.29-23.23; $\mathrm{p}<0.001$; 181365 days: HR, 7.67; 95\% CI, 2.34-22.80; $\mathrm{p}<0.001 ;>365$ days: HR, 2.63; 95\% CI, 0.96-6.03; p < 0.055; Table 5).

Only chronic renal failure under hemodialysis was associated with increased risk of AMI within 1 year of TKA (adjusted [A] HR, 4.34; 95\% CI, 1.22-15.43; $\mathrm{p}=$ 0.023; adjusted [B] HR, 4.71; 95\% CI, 1.30-17.09; $\mathrm{p}=$ 0.019; Tables 3, 5). Diabetes (adjusted [A] HR, 1.48; 95\% CI, 0.77-2.86; $\mathrm{p}=0.244$; adjusted [B] HR, 1.49; 95\% CI, 0.77-2.9; p = 0.236; Tables 3, 5) and hypertension (adjusted [A] HR, 1.36; 95\% CI, 0.46-3.99; p = 0.577; adjusted [B] HR, 1.30; 95\% CI, 0.44-3.82; p = 0.637; Tables 3, 5) were not associated with increased AMI risk.
The patients who underwent TKA with no history of $\mathrm{AMI} /$ stent had a lower risk (adjusted HR, 0.92; 95\% CI, $0.86-0.99 ; \mathrm{p}=0.016$ ) of AMI within 1 year of the surgery than the population with no surgery or AMI/stent in the study period.

\section{Discussion}

AMI after TKA is devastating, sometimes with fatal complications. Several studies have reported the risk of postTKA AMI in patients with a previous episode of AMI. However, previous studies did not compare different situations of AMI such as AMI with a stent, AMI without a stent, and a stent alone. Although risk-stratification tools exist that can anticipate the likelihood of AMI after noncardiac surgical procedures like TKA [6], such stratification provides guidance for a stent or AMI only and do not suggest the risk in patients with AMI + stent. Such a differentiation of patients would provide additional guidance for surgeons as to when to operate on patients with AMI with and without a stent. Therefore, this study aimed to determine the risk of post-TKA AMI subsequent to a coronary event such as AMI and/or stent. The study has limitations inherent to a large administrative database. This was an observational study so the associations observed may not be causal. In this study, the ICD-9 codes were used, which may lead to underreporting of comorbidities. It is 
Table 3. Effects of previous stent or AMI on subsequent AMI within 1 year of TKA (adjusted Model A)

\begin{tabular}{|c|c|c|c|}
\hline Variables & HR & $95 \% \mathrm{CI}$ & $\mathrm{p}$ value \\
\hline \multicolumn{4}{|c|}{ AMI/stent occurred before TKA } \\
\hline $\begin{array}{l}\text { No AMI/stent } \\
\text { (reference) }\end{array}$ & 1 & & \\
\hline Stent & 3.16 & $1.29-7.71$ & 0.012 \\
\hline AMI & 4.88 & $1.49-16.01$ & 0.009 \\
\hline $\mathrm{AMI}+$ stent & 5.23 & $1.81-15.14$ & 0.002 \\
\hline \multicolumn{4}{|l|}{ Gender } \\
\hline Female (reference) & 1 & & \\
\hline Male & 1.40 & $0.73-2.72$ & 0.315 \\
\hline \multicolumn{4}{|l|}{ Age (years) } \\
\hline$<65$ (reference) & 1 & & \\
\hline $65-69$ & 0.92 & $0.29-2.94$ & 0.893 \\
\hline $70-74$ & 1.18 & $0.41-3.43$ & 0.756 \\
\hline $75-79$ & 0.76 & $0.24-2.46$ & 0.646 \\
\hline$\geqq 80$ & 1.15 & $0.34-3.92$ & 0.827 \\
\hline \multicolumn{4}{|l|}{ Comorbidity } \\
\hline \multicolumn{4}{|l|}{ Hemodialysis } \\
\hline No (reference) & 1 & & \\
\hline Yes & 4.34 & $1.22-15.43$ & 0.023 \\
\hline \multicolumn{4}{|l|}{ Diabetes } \\
\hline No (reference) & 1 & & \\
\hline Yes & 1.48 & $0.77-2.86$ & 0.244 \\
\hline \multicolumn{4}{|l|}{ Hypertension } \\
\hline No (reference) & 1 & & \\
\hline Yes & 1.36 & $0.46-3.99$ & 0.577 \\
\hline
\end{tabular}

$\mathrm{AMI}=$ acute myocardial infarction; $\mathrm{HR}=$ hazard ratio; $\mathrm{CI}=$ confidence interval.

possible that coding errors may have affected the total percentage of AMI/stent. Moreover, the database does not contain detailed laboratory reports of patients. The limited number of variables available in the BNHI database makes it impossible to control other important confounders such as body mass index, lifestyle, exercise, or genetic factors.

The study suggests that any coronary artery event (stent, AMI, AMI + stent) increases the patient's risk of postTKA AMI within 1 year of the coronary event. ACC/AHA guidelines also suggest that the risk of post-TKA AMI increases with AMI or a stent [6]. However, this study also finds that patients having AMI + stent have a higher risk of post-TKA AMI compared with patients having only AMI or only a stent. After 6 months of AMI/stent, the risk was still high in the patients with a history of AMI without a stent. Between 6 months and 1 year, patients with AMI + a stent did not show a difference in risk compared with the reference. However, it is difficult to make safety recommendations based on this observation because the CI was very wide. The overall percentage of post-TKA AMI found
Table 4. Stratified analysis: hazard ratios of occurrence of AMI (365 days after surgery) for patients undergoing TKA

\begin{tabular}{lllr}
\hline Occurrence of AMI & HR & CI & p value \\
\hline No AMI/stent & 1 & & \\
$>365$ days & & & 0.293 \\
Stent & 1.67 & $0.71-3.94$ & 0.412 \\
AMI & 1.88 & $0.42-8.49$ & 0.321 \\
AMI + stent & 1.91 & $0.53-6.89$ & \\
181-365 days & & & 0.065 \\
Stent & 3.33 & $0.93-11.95$ & 0.001 \\
AMI & 18.27 & $3.97-84.15$ & \\
AMI + stent & 7.72 & $0.99-59.98$ & 0.003 \\
0-180 days & & & 0.010 \\
Stent & 4.98 & $1.72-14.38$ & $<0.001$ \\
AMI & 16.11 & $1.96-132.22$ & \\
AMI + stent & 27.27 & $7.52-98.94$ & \\
\hline AMI $=$ acute myocardial infarction; HR $=$ hazard ratio; CI $=$ confi- \\
dence interval.
\end{tabular}

Table 5. Risk of AMI based on TKA timing after initial AMI or stent (adjusted Model [B])

\begin{tabular}{|c|c|c|c|}
\hline Variables & HR & $95 \% \mathrm{CI}$ & $\mathrm{p}$ value \\
\hline \multicolumn{4}{|c|}{ Period between AMI/stent and TKA } \\
\hline $\begin{array}{l}\text { No AMI/stent } \\
\text { (reference) }\end{array}$ & 1 & & \\
\hline$>365$ day & 2.43 & $0.97-6.07$ & 0.058 \\
\hline 181-365 day & 7.52 & $2.47-22.88$ & $<0.001$ \\
\hline $0-180$ day & 8.42 & $3.03-23.41$ & $<0.001$ \\
\hline \multicolumn{4}{|l|}{ Gender } \\
\hline Female (reference) & 1 & & \\
\hline Male & 1.47 & $0.76-2.83$ & 0.256 \\
\hline \multicolumn{4}{|l|}{ Age (years) } \\
\hline$<65$ (reference) & 1 & & \\
\hline $65-69$ & 1.04 & $0.32-3.33$ & 0.952 \\
\hline $70-74$ & 1.36 & $0.46-3.96$ & 0.579 \\
\hline $75-79$ & 0.99 & $0.30-3.24$ & 0.986 \\
\hline$\geqq 80$ & 1.34 & $0.39-4.67$ & 0.644 \\
\hline \multicolumn{4}{|l|}{ Comorbidity } \\
\hline \multicolumn{4}{|l|}{ Hemodialysis } \\
\hline No (reference) & 1 & & \\
\hline Yes & 4.71 & $1.30-17.09$ & 0.019 \\
\hline \multicolumn{4}{|l|}{ Diabetes } \\
\hline No (reference) & 1 & & \\
\hline Yes & 1.49 & $0.77-2.90$ & 0.236 \\
\hline \multicolumn{4}{|l|}{ Hypertension } \\
\hline No (reference) & 1 & & \\
\hline Yes & 1.30 & $0.44-3.82$ & 0.637 \\
\hline
\end{tabular}

$\mathrm{AMI}=$ acute myocardial infarction; $\mathrm{HR}=$ hazard ratio $; \mathrm{CI}=$ confidence interval. 
Table 6. Comparison of results with those from the Danish population

\begin{tabular}{llc}
\hline $\begin{array}{l}\text { Interval between previous episode of } \\
\text { AMI/stent implantation and the TKA }\end{array}$ & Danish population (HR [CI]) & Taiwan population (HR [CI]) \\
\hline$<6$ months & $4.14(0.91-18.87)(\leq 6$ weeks $)$ & $8.42(3.03-23.41)$ \\
& $2.55(0.34-19.16)(6-52$ weeks $)$ & $7.22(2.47-22.88)$ \\
$6-12$ months & $2.18(0.28-16.79)(\leq 6$ weeks $)$ & $2.43(0.97-6.07)$ \\
& $0.96(0.43-2.17)(\leq 6$ weeks $)$ & \\
\hline
\end{tabular}

$\mathrm{AMI}=$ acute myocardial infarction; $\mathrm{HR}=$ hazard ratio; $\mathrm{CI}=$ confidence interval.

in this study falls within the range of the percentage from most of the previous studies $[7,8]$ and was similar to that reported in the Danish study [12]. Some studies have reported post-TKA AMI between $0.1 \%$ and $1.8 \%[8,11$, 13 ], which falls out of the range of the majority of studies. Such conflicting results have been suggested to be attributable to differences in the criteria for diagnosing AMI [12]. The majority of the studies have investigated AMI within 90 days of TKA, whereas the Danish study population included cases for 12 months. Although this study also included such cases, there was a higher risk of AMI in the Taiwan population compared with the Danish population (Table 6) [12]. Such disparities may arise as a result of differences in patient characteristics and the centers where the surgeries were performed.

This study suggests that the risk of post-TKA AMI decreases with the time interval between AMI and TKA; however, it is greatest within 6 months after AMI. The patients with a history of AMI + stent had the highest risk of post-TKA AMI compared with those with only AMI or only a stent within 6 months of AMI. ACC/AHA guidelines also suggest that the risk of AMI decreases with the increase in the time interval between AMI and TKA [6]. In addition, our study shows how that risk would vary if the patient is having a stent alone, AMI with a stent, or AMI without a stent. Stents are expected to provide protection against postoperative AMI in noncardiac surgeries [14]; however, patients with AMI + a stent had a high risk of post-TKA AMI within 1 year of its placement. Previous studies to evaluate the risk of postoperative catastrophic events in the patients with a stent have reported variable recommendations about timing for surgery [10, 19, 24]. The high risk has been attributed to the discontinuation of antiplatelet drugs before the surgery [10, 19, 24]. This high risk also suggests that the timing of the TKA for some patients with AMI + stent did not comply with the timing (after 6 months) recommended by ACC/AHA for patients after percutaneous coronary intervention. The Danish study also suggests that the percentage of post-TKA AMI decreases with the increase in the interval between AMI and TKA. However, they reported a higher HR than in our study (Table 6). The difference observed between the
Taiwan and the Danish populations may be attributed to the differences in the postsurgical time interval and the matched control patients of the two studies. The matched control for the Danish studies comprised patients who underwent surgery other than TKA, whereas our matched control group consisted of patients who underwent TKA and had no history of AMI/stent.

This study found a higher risk of AMI after TKA in patients on hemodialysis. This is in accordance with other studies and ACC/AHA guidelines [6], in which a higher risk of myocardial infarction has been reported in patients on renal failure. A high mortality rate ratio [18] resulting from myocardial infarction within the 3 years of hemodialysis and a high AMI incidence [20] in hemodialysis patients have been reported in previous studies. The higher risk of AMI in hemodialysis patients has been attributed to the increased probability of blood clot formation as a result of coagulation activation, depletion of platelet granules, and endothelial integrity [21].

The lower risk of AMI in patients who had TKA and no history of AMI compared with the patients with no TKA corroborates with other studies, which compared the life expectancy of patients undergoing THA with the general population [9, 23]. This may be the result of improved mobilization and/decreased pain beneficial to the cardiovascular health status of the patients.

This study found that the risk of post-TKA AMI is highest within 6 months of the previous AMI episode. This risk remains high up to 1 year post-AMI but normalizes thereafter. Stents do not provide protection against postTKA AMI within 6 months of an AMI and the risk also remains high up to 1 year. Furthermore, hemodialysis patients also have a very high risk of post-TKA AMI. However, the risk of AMI in patients undergoing TKA within 1 year of TKA is lower than the general population. In conclusion, it is recommended that an elective TKA is performed at least 1 year after an episode of $\mathrm{AMI} / \mathrm{stent}$ placement.

Acknowledgments We thank Dr Chien-Cheng Chen (Department of Cardiology and Medicine, Show Chwan Memorial Hospital, Taiwan) for providing a cardiologist's view of the results. We also thank 
Dr Janet Huang (Chief Operation Officer, Show Chwan Healthcare System, Taiwan) for helping us procure the BNHI data.

\section{References}

1. Badner NH, Knill RL, Brown JE, Novick TV, Gelb AW. Myocardial infarction after noncardiac surgery. Anesthesiology. 1998;88:572-578.

2. Cheng CL, Kao YH, Lin SJ, Lee CH, Lai ML. Validation of the National Health Insurance Research Database with ischemic stroke cases in Taiwan. Pharmacoepidemiol Drug Saf. 2011;20:236-242.

3. Cheng CL, Lee CH, Chen PS, Li YH, Lin SJ, Yang YH. Validation of acute myocardial infarction cases in the national health insurance research database in Taiwan. $J$ Epidemiol. 2014;24:500-507.

4. Devereaux PJ, Goldman L, Cook DJ, Gilbert K, Leslie K, Guyatt GH. Perioperative cardiac events in patients undergoing noncardiac surgery: a review of the magnitude of the problem, the pathophysiology of the events and methods to estimate and communicate risk. CMAJ. 2005;173:627-634.

5. Feinglass J, Amir H, Taylor P, Lurie I, Manheim LM, Chang RW. How safe is primary knee replacement surgery? Perioperative complication rates in Northern Illinois, 1993-1999. Arthritis Rheum. 2004;51:110-116.

6. Fleisher LA, Fleischmann KE, Auerbach AD, Barnason SA, Beckman JA, Bozkurt B, Davila-Roman VG, Gerhard-Herman MD, Holly TA, Kane GC, Marine JE, Nelson MT, Spencer CC, Thompson A, Ting HH, Uretsky BF, Wijeysundera DN. 2014 ACC/AHA Guideline on Perioperative Cardiovascular Evaluation and Management of Patients Undergoing Noncardiac Surgery: Executive Summary: a report of the American College of Cardiology/American Heart Association Task Force on Practice Guidelines. J Am Coll Cardiol. 2014;64:2373-2405.

7. Fox KA, Dabbous OH, Goldberg RJ, Pieper KS, Eagle KA, Van de Werf F, Avezum A, Goodman SG, Flather MD, Anderson FA, Jr., Granger CB. Prediction of risk of death and myocardial infarction in the six months after presentation with acute coronary syndrome: prospective multinational observational study (GRACE). BMJ (Clin Res Ed). 2006;333:1091.

8. Gandhi R, Petruccelli D, Devereaux PJ, Adili A, Hubmann M, de Beer J. Incidence and timing of myocardial infarction after total joint arthroplasty. J Arthroplasty. 2006;21:874-877.

9. Garellick G, Malchau H, Herberts P, Hansson E, Axelsson H, Hansson T. Life expectancy and cost utility after total hip replacement. Clin Orthop Relat Res. 1998;346:141-151.

10. Kaluza GL, Joseph J, Lee JR, Raizner ME, Raizner AE. Catastrophic outcomes of noncardiac surgery soon after coronary stenting. J Am Coll Cardiol. 2000;35:1288-1294.

11. Khatod M, Inacio M, Paxton EW, Bini SA, Namba RS, Burchette RJ, Fithian DC. Knee replacement: epidemiology, outcomes, and trends in Southern California: 17,080 replacements from 1995 through 2004. Acta Orthop. 2008;79:812-819.
12. Lalmohamed A, Vestergaard P, Klop C, Grove EL, de Boer A, Leufkens HG, van Staa TP, de Vries F. Timing of acute myocardial infarction in patients undergoing total hip or knee replacement: a nationwide cohort study. Arch Intern Med. 2012;172:1229-1235.

13. Liu SS, Bae JJ, Bieltz M, Ma Y, Memtsoudis S. Association of perioperative use of nonsteroidal anti-inflammatory drugs with postoperative myocardial infarction after total joint replacement. Reg Anesth Pain Med. 2012;37:45

14. Livhits M, Gibbons MM, de Virgilio C, O'Connell JB, Leonardi MJ, Ko CY, Zingmond DS. Coronary revascularization after myocardial infarction can reduce risks of noncardiac surgery. $J$ Am Coll Surg. 2011;212:1018-1026.

15. Mantilla CB, Horlocker TT, Schroeder DR, Berry DJ, Brown DL. Frequency of myocardial infarction, pulmonary embolism, deep venous thrombosis, and death following primary hip or knee arthroplasty. Anesthesiology. 2002;96:1140-1146.

16. Mantilla CB, Wass CT, Goodrich KA, Johanns CJ, Kool ML, Zhu X, Corredor JA, Warner DO, Joyner MJ, Berry DJ, Schroeder DR, Sprung J. Risk for perioperative myocardial infarction and mortality in patients undergoing hip or knee arthroplasty: the role of anemia. Transfusion. 2011;51:82-91.

17. Ministry of Health and Welfare, Taiwan. National health insurance health statistics annual report. Available at: http://www. mohw.gov.tw/cht/DOS/Statistic.aspx?f_list_no=312\&fod_list_no $=2680$. Accessed August 13, 2013.

18. Ocak G, van Stralen KJ, Rosendaal FR, Verduijn M, Ravani P, Palsson R, Leivestad T, Hoitsma AJ, Ferrer-Alamar M, Finne P, De Meester J, Wanner C, Dekker FW, Jager KJ. Mortality due to pulmonary embolism, myocardial infarction, and stroke among incident dialysis patients. J Thromb Haemost. 2012;10:24842493.

19. Reddy PR, Vaitkus PT. Risks of noncardiac surgery after coronary stenting. Am J Cardiol. 2005;95:755-757.

20. Sanchez-Perales C, Vazquez-Ruiz de Castroviejo E, SeguraTorres P, Borrego-Utiel F, Garcia-Cortes MJ, Garcia-Garcia F, Biechy-Baldan MM, Gil-Cunquero JM, Liebana-Canada A. Incidence of acute myocardial infarction in the evolution of dialysis patients. Nefrologia. 2012;32:597-604.

21. Schoorl M, Schoorl M, Nube MJ, Bartels PC. Coagulation activation, depletion of platelet granules and endothelial integrity in case of uraemia and haemodialysis treatment. BMC Nephrol. 2013;14:72.

22. Shah KB, Kleinman BS, Sami H, Patel J, Rao TL. Reevaluation of perioperative myocardial infarction in patients with prior myocardial infarction undergoing noncardiac operations. Anesth Analg. 1990;71:231-235.

23. Visuri T, Pulkkinen P, Turula KB, Paavolainen P, Koskenvuo M. Life expectancy after hip arthroplasty. Case-control study of 1018 cases of primary arthrosis. Acta Orthop Scand. 1994;65:9-11.

24. Wijeysundera DN, Wijeysundera HC, Yun L, Wasowicz M, Beattie WS, Velianou JL, Ko DT. Risk of elective major noncardiac surgery after coronary stent insertion: a population-based study. Circulation. 2012;126:1355-1362. 